\title{
GAMES ORBITS PLAY AND OBSTRUCTIONS TO BOREL REDUCIBILITY
}

\author{
MARTINO LUPINI AND ARISTOTELIS PANAGIOTOPOULOS
}

\begin{abstract}
We introduce a new game-theoretic approach to anti-classification results for orbit equivalence relations. Within this framework, we give a short conceptual proof of Hjorth's turbulence theorem. We also introduce a new dynamical criterion providing an obstruction to classification by orbits of CLI groups. We apply this criterion to the relation of equality of countable sets of reals, and the relations of unitary conjugacy of unitary and selfadjoint operators on the separable infinite-dimensional Hilbert space.
\end{abstract}

\section{INTRODUCTION}

Classification problems arise naturally in many areas of mathematics. Consider for example the problem of classifying all bounded selfadjoint operators $T \in \mathcal{B}(\mathcal{H})_{\mathrm{sa}}$ on a Hilbert space $\mathcal{H}$ up to unitary equivalence. If $\mathcal{H}$ is of finite dimension $n$, then we can assign to every selfadjoint operator $T$ the tuple $f(T)=\left(\lambda_{0}, \ldots, \lambda_{n-1}\right)$ of its increasingly ordered eigenvalues, counting repetition. The map $f: \mathcal{B}(\mathcal{H})_{\mathrm{sa}} \rightarrow \mathbb{R}^{n}$ has the property that $T, S$ are unitarily equivalent if and only if $f(T)=f(S)$. Moreover, since $f$ is computed by an explicit formula, one can recover the relation of unitary conjugacy of selfadjoint operators from the relation of equality on $\mathbb{R}^{n}$. The same is far from being true when $\mathcal{H}$ is infinite dimensional. In this case, there is no Borel map $f: \mathcal{B}(\mathcal{H})_{\mathrm{sa}} \rightarrow Y$, where $Y$ is a Polish space, so that $T, S$ are unitarily conjugate if and only if $f(T)=f(S)$; see [6]. The situation is actually much worse, as we discuss below.

Let $E, F$ be two equivalence relations on a Polish spaces $X, Y$, respectively. A $(E, F)$-homomorphism is a map $f: X \rightarrow Y$ with the property $x E y \Rightarrow f(x) E f(y)$. A $(E, F)$-homomorphism $f$ is a reduction from $E$ to $F$, if $x E y \Leftrightarrow f(x) E f(y)$. Given $E, F$ as above we are interested in the problem of whether there is a reduction $f$ from $E$ to $F$ that is moreover Borel as a map from $X$ to $Y$. In this case, $E$ is said to be Borel reducible to $F$. Recall that $E$ is concretely classifiable if $E$ is Borel reducible to equality on some Polish space $Y$. One can associate to every countable language $\mathcal{L}$ the $\operatorname{Polish}$ space $\operatorname{Mod}(\mathcal{L})$ of all $\mathcal{L}$-structures with domain $\mathbb{N}$. For $x, y \in \operatorname{Mod}(\mathcal{L})$ we write $x \cong_{\mathcal{L}} y$ if $x, y$ are isomorphic $\mathcal{L}$-structures. An equivalence relation $E$ on a Polish space $X$ is classifiable by countable structures if it is Borel reducible to $\cong_{\mathcal{L}}$ for some countable language $\mathcal{L}$. As we noted above the relation of unitary conjugacy of bounded selfadjoint operators on an infinite-dimensional Hilbert space is not concretely classifiable. This result was then strengthened by Kechris and Sofronidis [25], who proved that such a relation is not classifiable by countable structures. The same conclusions hold for unitary operators.

Particularly interesting is the case when the equivalence relation $E$ on $X$ is obtained as the orbit equivalence relation $E_{G}^{X}$ of a continuous action of a Polish group $G$ on $X$. In particular, all examples mentioned so far are of this form. For instance, unitary equivalence of selfadjoint operators is induced by the action of the unitary group $\mathcal{U}(\mathcal{H})$ on $\mathcal{B}(\mathcal{H})_{\text {sa }}$ by conjugation. Similarly, the relation $\cong_{\mathcal{L}}$ of isomorphism of $\mathcal{L}$-structures is induced by the canonical logic action of $S_{\infty}$ on $\operatorname{Mod}(\mathcal{L})$. Finally, the equality relation on a Polish space is induced by the action of the trivial group. Both aforementioned anti-classification results stem from the careful study of which restrictions the topology on $G$ puts on a $G$-space $X$. To be precise, consider the following general problem.

Problem 1.1. Given a class of Polish groups $\mathcal{C}$, which dynamical conditions on a Polish $G$-space $X$ ensure that the corresponding orbit equivalence relation is not Borel reducible to $E_{H}^{Y}$ for some Borel action of a Polish group $H$ in $\mathcal{C}$ on a Polish space $Y$ ?

By [4, Corollary 5.1.6], in Problem 1.1 one can assume without loss of generality that the action of $H$ on $Y$ is continuous. The property of having meager orbits and a dense orbit provides such a criterion for the class $\mathcal{C}$ of compact Polish groups [12, Proposition 6.1.10]. This is used in [6] to prove that bounded selfadjoint operators on an infinite-dimensional Hilbert space are not concretely classifiable up to unitary conjugacy. Hjorth's turbulence

2000 Mathematics Subject Classification. Primary 03E15, 54H20; Secondary 20L05, $54 \mathrm{H} 05$.

Key words and phrases. CLI group, Polish group, Polish groupoid, turbulence, orbit equivalence relation, Borel game.

This work was initiated during a visit of A.P. at the California Institute of Technology in the Spring 2016. The authors gratefully acknowledge the hospitality and the financial support of the Institute. M.L. was partially supported by the NSF Grant DMS-1600186. 
theory, initially developed by Hjorth in [16], addresses this problem in the case when $\mathcal{C}$ is the class of nonArchimedean Polish groups. Turbulence is used in [25] to prove that bounded selfadjoint operators on an infinitedimensional Hilbert space are not classifiable by countable structures up to unitary conjugacy. Turbulence has played a key role in Borel complexity theory in the last two decades and it is to this day essentially the only known method to prove unclassifiability by countable structures; see $[1,2,7-9,12-14,16,19,21,23,24,26,27,29,35-38]$. There has been so far little progress into obtaining similar criteria for other interesting classes of Polish groups.

The purpose of this paper is two-fold. Our first goal is to introduce a game-theoretic approach to Problem 1.1. This approach consists in endowing the space $X / G$ of orbits of a Polish $G$-space $X$ with different graph structures, and then showing that a Baire measurable $\left(E_{G}^{X}, E_{H}^{Y}\right)$-homomorphism $f: X \rightarrow Y$ induces a graph homomorphism $X / G \rightarrow Y / G$ after restricting to an invariant dense $G_{\delta}$ set. This perspective allows us to give a short conceptual proof of Hjorth's turbulence theorem, avoiding the substantial amount of bookkeeping of Hjorth's original argument [16]; see also [12, Chapter 10].

The second goal of this paper is to use the above-mentioned game-theoretic approach to address Problem 1.1 for the class of CLI groups. Recall that a CLI group is a Polish group that admits a compatible left-invariant metric. Every locally compact group, as well as every solvable Polish group - in particular, every abelian Polish group -is CLI [18, Corollary 3.7]. Such a class of groups has been considered in several papers so far. For instance, [3, Corollary 5.C.6] settled the topological Vaught conjecture for CLI groups. It is also proved in [3, Theorem 5.B.2] that CLI groups satisfy an analog of the Glimm-Effros dichotomy. In [11, Theorem 1.1] it is shown that the non-Archimedean CLI groups are precisely the automorphism groups of countable structures whose Scott sentence does not have an uncountable model. The class of CLI groups has been further studied in [30], where it is shown that if forms a coanalytic non-Borel subset of the class of Polish groups.

A fundamental tool in the study of dichotomies for orbit equivalence relations from [3] is the notion of $\iota$-embeddability — which we call Becker embeddability - for points in a Polish $G$-space. We prove that a Bairemeasurable homomorphism between orbit equivalence relations necessarily preserves Becker embeddability on an invariant dense $G_{\delta}$ set. From this we extract in Theorem 2.9 a dynamical condition which answers Problem 1.1 for the class of CLI groups. We then apply it to show that the Friedman-Stanley jump of equality $=^{+}$is not Borel reducible to the orbit equivalence relation induced by a Borel action of a CLI group. The only proof of this fact that we are aware of relies on meta-mathematical reasoning and involves the theory of pinned equivalence relations; see [20]. A natural reduction from this relation to the relations of unitary equivalence of bounded unitary or selfajdoint operators on an infinite-dimensional Hilbert space, shows that the latter relations are also not classifiable by the orbits of a CLI group actions. We note that it is still an open question whether the unitary group $\mathcal{U}(\mathcal{H})$ of the separable infinite dimensional Hilbert space $\mathcal{H}$ can produce under some action on a Polish space an orbit equivalence relation that is universal for orbit equivalence relations induced by continuous Polish group actions. Our results show that the complexity of possible orbit equivalence relations of $\mathcal{U}(\mathcal{H})$-actions is not bounded from above by the complexity of orbit equivalence relations induced by continuous CLI group actions.

We conclude by discussing how all the results of the present paper admit natural generalizations from Polish group actions to Polish groupoids. Turbulence theory for Polish groupoids has been developed in [13]. Applications of this more general framework to classification problems in operator algebras have also been presented in [13].

Besides this introduction, the present paper is divided into three sections. In Section 2 we present the results about Becker-embeddability and CLI groups. In Section 3 we present the short and conceptual proof of Hjorth's turbulence theorem mentioned above. Finally in Section 4 we recall the fundamental notions about Polish groupoids, and explain how the main results of this paper can be adapted to this more general setting.

Acknowledgments. We are grateful to Samuel Coskey, Alexander Kechris, and Sławomir Solecki for many helpful suggestions and remarks.

\section{Nonreducibility to CLI GRoup aCtions}

2.1. The Becker-embedding game. Recall that a CLI group is a Polish group that admits a compatible complete left-invariant metric. It is easy to see that a Polish group is CLI if and only if it admits a compatible right-invariant metric; see [3, 3.A.2. Proposition]. Suppose that $G$ is a Polish group, and $X$ is a Polish $G$ space. The main goal of this section is to provide a dynamical criterion of a Polish $G$-space $X$ to show that the corresponding orbit equivalence relation is not Borel reducible to the orbit equivalence relation induced by a Borel action of a CLI group.

We recall the notion of $\iota$-embeddability for points of $X$ from [3, Definition 3.D.1]. 
Definition 2.1. Let $d$ be a left-invariant metric on $G$. If $x, y \in X$, then $x$ is $\iota$-embeddable into $y$ if there exists a sequence $\left(h_{n}\right)_{n \in \omega}$ in $G$ such that $h_{n} x \rightarrow y$ and $\left(h_{n}\right)_{n \in \omega}$ is Cauchy with respect to the metric $d$.

By [3, Proposition 3.B.1] the definition does not depend on the choice of the left-invariant metric $d$ on $G$. We now consider a natural game between two players, and show that it captures the notion of $\iota$-embeddability from Definition 2.1.

Definition 2.2. Suppose that $X$ is a Polish $G$-space, and $x, y \in X$. We consider the Becker-embedding game $\operatorname{Emb}(x, y)$ played between two players as follows. Set $U_{0}=X$ and $V_{0}=G$.

(1) In the first turn, Player I plays an open neighborhood $U_{1}$ of $x$, and an open neighborhood $V_{1}$ of the identity of $G$. Player II replies with an element $g_{0}$ in $V_{0}$.

(2) In the second turn, Player I then plays an open neighborhood $U_{2}$ of $x$, and an open neighborhood $V_{2}$ of the identity of $G$, and Player II replies with an element $g_{1}$ in $V_{1}$.

(n) At the $n$-th turn, Player I plays an open neighborhood $U_{n}$ of $x$, and an open neighborhood $V_{n}$ of the identity of $G$, and Player II responds with an element $g_{n-1}$ in $V_{n-1}$.

The game proceed in this way, producing a sequence $\left(g_{n}\right)$ of elements of $G$, a sequence $\left(U_{n}\right)$ of open neighborhoods of $x$ in $X$, and a sequence $\left(V_{n}\right)$ of open neighborhoods of the identity in $G$. Player II wins the game if for every $n>0, g_{n-1} \cdots g_{0} y \in U_{n}$. We say that $x$ is Becker embeddable into $y$-and write $x \preccurlyeq \mathcal{B} y$-if Player II has a winning strategy for the game $\operatorname{Emb}(x, y)$.

Remark 2.3. It is not difficult to see that, if Player II has a winning strategy for the Becker-embedding game as described in Definition 2.2, then it also has a winning strategy for the same game with the additional winning conditions that $g_{n}$ belongs to some given comeager subset of $V_{n}$, and $g_{n-1} \cdots g_{0} y$ belongs to some given comeager subset $X_{0}$ of $X$, provided that the set of $g \in G$ such that $g y \in X_{0}$ is comeager. This is a consequence of the following version of the Kuratowski-Ulam theorem: suppose that $X, Y$ are Polish spaces and $f: X \rightarrow Y$ is a continuous open map. Then a Baire-measurable subset $A$ of $X$ is comeager if and only if the set $\left\{y \in Y: A \cap f^{-1}\{y\}\right.$ is comeager in $\left.f^{-1}\{y\}\right\}$ is comeager; see [31, Theorem A.1]. One can then apply this fact to the continuous and open map $G \times X \rightarrow X,(g, x) \mapsto g x$. This observation can be equivalently phrased in terms of properties of the Vaught transform for Polish group actions; see [12, Section 3.2].

We now show that the notion of Becker-embeddability from Definition 2.2 is actually equivalent to the notion of $\iota$-embeddability from Definition 2.1.

Lemma 2.4. Let $X$ be a Polish $G$-space. If $x, y$ are points of $X$, then the following statements are equivalent:

(1) $x \preccurlyeq \mathcal{B} y$;

(2) $x$ is $\iota$-embeddable in $y$.

Proof. We fix a left-invariant metric $d$ on $G$. For a subset $A$ of $G$ we let $\operatorname{diam}(A)$ be the $\operatorname{diameter}$ of $A$ with respect to $d$.

$(1) \Rightarrow(2)$ Suppose that Player II has a winning strategy for the Becker-embedding game Emb $(x, y)$. Let Player I play a sequence $\left(U_{n}\right)$ which forms a basis of open neighborhoods of $x$ and a sequence $\left(V_{n}\right)$ which forms a basis of symmetric open neighborhoods of the identity of $G$ with $\operatorname{diam}\left(V_{n}\right)<2^{-n}$. Let $\left(g_{n}\right)_{n \in \omega}$ be the sequence of elements of $G$ given by a winning strategy for Player II. Then the sequence $\left(h_{n}\right)_{n \in \omega}$ obtained by setting $h_{n}:=g_{0}^{-1} \cdots g_{n}^{-1}$ is $d$-Cauchy, and $h_{n} x \rightarrow y$.

$(2) \Rightarrow(1)$ Suppose that there exists a $d$-Cauchy sequence $\left(h_{n}\right)_{n \in \omega}$ in $G$ such that $h_{n} x \rightarrow y$. We describe a winning strategy for Player II. Set $h_{-1}:=1$. Suppose that in the first turn Player I plays an open neighborhood $U_{1}$ of $x$ and an open neighborhood $V_{1}$ of the identity of $G$. Player II replies with $g_{0}:=h_{k_{0}}^{-1}$, where $k_{0} \in \omega$ is such so that:

(1) $h_{k}^{-1} h_{k_{0}} \in V_{1}$ for all $k \geq k_{0}$, and

(2) $h_{k_{0}}^{-1} y \in U_{1}$.

The first condition is satisfied by a large enough $k_{0} \in \omega$ because $\left(h_{n}\right)_{n \in \omega}$ is $d$-Cauchy. The second condition is satisfied by a large enough $k_{0} \in \omega$ because $h_{n} x \rightarrow y$. Recursively, suppose that in the $n$-turn Player I plays an open neighborhood $U_{n}$ of $x$ and an open neighborhood $V_{n}$ of the identity in $G$. Inductively, assume also that $g_{n-1}$ is of the form $h_{k_{n-2}}^{-1} h_{k_{n-3}}$ for some $k_{n-2} \in \omega$ such that $h_{k}^{-1} h_{k_{n-2}} \in V_{n-1}$ for all $k \geq k_{n-2}$. Player II replies with $g_{n-1}:=h_{k_{n-1}}^{-1} h_{k_{n-2}}$, where $k_{n-1} \in \omega$ is such that:

(1) $h_{k}^{-1} h_{k_{n-1}} \in V_{n}$ for all $k \geq k_{n-1}$, and

(2) $h_{k_{n-1}}^{-1} y \in U_{n}$. 
Again, our assumptions on the sequence $\left(h_{n}\right)_{n \in \omega}$ guarantee that a large enough $k_{n-1} \in \omega$ satisfies both these conditions. Then we have that $g_{n-1} \in V_{n-1}$ by the inductive assumption on $k_{n-2}$. Therefore this procedure describes a winning strategy for Player II in the Becker-embedding game $\operatorname{Emb}(x, y)$.

Suppose that $X$ is a Polish $G$-space. It follows from Lemma 2.4 and [3, Proposition 3.D.4] that the relation of Becker-embeddability is a preorder. Furthermore, if $x \preccurlyeq \mathcal{B} y, x^{\prime}$ belongs to the $G$-orbit of $x$, and $y^{\prime}$ belongs to the $G$-orbit of $y$, then $x^{\prime} \preccurlyeq \mathcal{B} y^{\prime}$.

We let $X / G$ be the space of $G$-orbits of points of $X$. The Becker-embeddability preorder defines a directed graph structure on $X / G$ obtained by declaring that there is an arrow from the orbit $[x]$ of $x$ to the orbit $[y]$ of $y$ if and only if $x \preccurlyeq \mathcal{B} y$. We will call this the Becker digraph $\mathcal{B}(X / G)$ of the Polish $G$-space $X$. Similarly, for a $G$-invariant subset $X_{0}$ of $X$ we let $\mathcal{B}\left(X_{0} / G\right)$ the induced subgraph of $\mathcal{B}(X / G)$ only containing vertices corresponding to orbits from $X_{0}$. Suppose that $G, H$ are Polish groups, $X$ is a Polish $G$-space, and $Y$ is a Polish $H$-space. Any $\left(E_{G}^{X}, E_{H}^{Y}\right)$-homomorphism $f: X \rightarrow Y$ induces a function $[f]: X / G \rightarrow Y / H,[x] \mapsto[f(x)]$. We will show below that, when $f$ is Baire-measurable, such a function is generically a digraph homomorphism with respect to the Becker digraph structures on $X / G$ and $Y / H$.

We now recall an example from [3], describing the notion of Becker-embedding in case of Polish $G$-spaces arising from classes of countable models. Suppose that $\mathcal{L}=\left(R_{i}\right)_{i \in I}$ is a countable first order relational language, where $R_{i}$ is a relation symbol with arity $n_{i}$. Let $\operatorname{Mod}(\mathcal{L})$ be the space of countable $\mathcal{L}$-structures having $\mathbb{N}$ as support, $F$ be a countable fragment of $\mathcal{L}_{\omega_{1}, \omega}$, and $S_{\infty}$ be the group of permutations of $\mathbb{N}$. As usual, one can regard $\operatorname{Mod}(\mathcal{L})$ as the product $\prod_{i \in I} 2^{\left(\mathbb{N}^{n_{i}}\right)}$. Any $\mathcal{L}_{\omega_{1}, \omega}$ formula $\varphi$ with parameters from $\mathbb{N}$ defines a subset $[\varphi]$ of $\operatorname{Mod}(\mathcal{L})$ of the structures that satisfy $\varphi$. The fragment $F$ defines a topology $t_{F}$ on $\operatorname{Mod}(\mathcal{L})$ having the collections of sets of the form $[\varphi]$, where $\varphi$ ranges among the formulas in $F$ with parameters, as a clopen basis. The canonical action $S_{\infty} \curvearrowright \operatorname{Mod}(\mathcal{L})$ turns $\left(\operatorname{Mod}(\mathcal{L}), t_{F}\right)$ into a Polish $G$-space [3, Proposition 2.D.2]. If $x, y \in \operatorname{Mod}(\mathcal{L})$ then $x \preccurlyeq \mathcal{B} y$ if and only if there exists an injective function $f: \mathbb{N} \rightarrow \mathbb{N}$ that represents an $F$ embedding from $x$ to $y$. This means that $f$ preserves the value of formulas $\varphi$ in $F$ with parameters [3, Proposition 3.D.2]. In the particular case when $F$ is the fragment consisting of atomic first-order formulas, the topology $t_{F}$ coincides with the product topology, and an $F$-embedding is the same as an embedding as $\mathcal{L}$-structure. When $F$ is the fragment consisting of all first-order formulas, an $F$-embedding is an elementary embedding.

2.2. The orbit continuity lemma. Recall that if $E, F$ are equivalence relations on Polish spaces $X, Y$ respectively, then a $(E, F)$-homomorphism is a function $f: X \rightarrow Y$ mapping $E$-classes to $F$-classes. In this subsection we isolate a lemma to be used in the rest of the paper. It states that a Baire-measurable homomorphism between orbit equivalence relations admits a restriction to a dense $G_{\delta}$ set which is continuous at the level of orbits, in a suitable sense. Variations of such a lemma are well known. The starting point is essentially [16, Lemma 3.17] modified as in the beginning of the proof of [16, Theorem 3.18]; see also [12, Lemma 10.1.4 and Theorem 10.4.2].

Lemma 2.5. Suppose that $G, H$ are Polish groups, $X$ is a Polish $G$-space, and $Y$ is a Polish $H$-space. Let $f: X \rightarrow Y$ be a Baire-measurable $\left(E_{G}^{X}, E_{H}^{Y}\right)$-homomorphism. Then there exists a dense $G_{\delta}$ subset $C$ of $X$ such that

- the restriction of $f$ to $C$ is continuous;

- for any $x \in C,\{g \in G: g x \in C\}$ is a comeager subset of $G$;

- for any $x_{0} \in C$ and for any open neighborhood $W$ of the identity in $H$ there exists an open neighborhood $U$ of $x_{0}$ and an open neighborhood $V$ of the identity of $G$ such that for any $x \in U \cap C$ and for a comeager set of $g \in V$, one has that $f(g x) \in W f(x)$ and $g x \in C$.

Proof. In the course of the proof, we will use the category quantifier $\forall^{*} x \in U$ for the statement "for a comeager set of $x \in U^{\prime \prime}$; see [12, Section 3.2]. Fix a neighborhood $W_{0}$ of the identity in $H$. We first prove the following claim: $\forall x_{0} \in X \forall^{*} g_{0} \in G$, there is an open neighborhood $V$ of the identity in $G$ such that $\forall^{*} g_{1} \in V$, $f\left(g_{1} g_{0} x_{0}\right) \in W_{0} f\left(g_{0} x_{0}\right)$.

Fix a neighborhood $W$ of the identity of $H$ such that $W W^{-1} \subset W_{0}$. Let $\left(h_{n}\right)$ be a sequence in $H$ such that $\left\{W h_{n}: n \in \mathbb{N}\right\}$ is a cover of $H$. Since $W h_{n} f\left(x_{0}\right)$ is analytic, the set of elements $x$ of the orbit of $x_{0}$ such that $f(x) \in W h_{n} f\left(x_{0}\right)$ has the Baire property. Therefore we can find a sequence $\left(O_{n}\right)$ of open subsets of $G$ with dense union $O$ and a comeager subset $D$ of $O$ such that $\forall g \in D \cap O_{n}, f\left(g x_{0}\right) \in W h_{n} f\left(x_{0}\right)$. Suppose now that $g_{0} \in D$. Let $n \in \mathbb{N}$ be such that $g_{0} \in O_{n}$. Then there exists a neighborhood $V$ of the identity of $G$ such that $V g_{0} \subset O_{n}$. Observe that $\left(D \cap O_{n}\right) g_{0}^{-1} \cap V$ is a comeager subset of $V$. If $g_{1} \in\left(D \cap O_{n}\right) g_{0}^{-1} \cap V$, then we have

$$
f\left(g_{1} g_{0} x_{0}\right) \in W h_{n} f\left(x_{0}\right) \quad \text { and } \quad f\left(g_{0} x_{0}\right) \in W h_{n} f\left(x_{0}\right) .
$$


Therefore

This concludes the proof of the claim.

$$
f\left(g_{1} g_{0} x_{0}\right) \in W W^{-1} f\left(g_{0} x_{0}\right) \subset W_{0} f\left(g_{0} x_{0}\right) .
$$

From the claim and the Kuratowski-Ulam theorem, one deduces that there exists a dense $G_{\delta}$ subset $C_{0}$ of $X$ such that for every $x \in C_{0}$ there exists an open neighborhood $V$ of the identity of $G$ such that $\forall^{*} g \in V$, $f(g x) \in W f(x)$. Since $f$ is Baire-measurable, we can furthermore assume that the restriction of $f$ to $C_{0}$ is continuous.

Fix now a countable basis $\left(W_{k}\right)$ of open neighborhoods of the identity of $H$ and a countable basis $\left(V_{n}\right)$ of open neighborhoods of the identity in $G$. Let $N: X \times \mathbb{N} \rightarrow \mathbb{N} \cup\{\infty\}$ be the function that assigns to $(x, k)$ the least $n \in \mathbb{N}$ such that $\forall^{*} g \in V_{n}, f(g x) \in W_{k} f(x)$ if such an $n$ exists and $x \in C_{0}$, and $\infty$ otherwise. Then $N$ is an analytic function, and hence one can find a dense $G_{\delta}$ subset $C_{1}$ of $X$ contained in $C_{0}$ such that $\left.N\right|_{C_{1} \times \mathbb{N}}$ is continuous. By [12, Proposition 3.2.5 and Theorem 3.2.7] the set $C:=\left\{x \in C_{1}: \forall^{*} g \in G, g x \in C_{1}\right\}$ is a dense $G_{\delta}$ subset of $X$ such that $\forall x \in C, \forall^{*} g \in G, g x \in C$. Therefore $C$ satisfies the desired conclusions.

2.3. Generic homomorphisms between Becker graphs. In this section we use the Becker-embedding game and the orbit continuity lemma to address Problem 1.1 for the class of CLI groups.

Definition 2.6. An equivalence relation $E$ on a Polish space $X$ is CLI-classifiable if it is Borel reducible to $E_{H}^{Y}$ for some CLI group $H$ and Polish $H$-space $Y$.

We will obtain below an obstruction to CLI-classiability in terms of the Becker digraph. This will be based upon the following properties of the Becker digraph:

(1) the Becker digraph contains only loops in the case of CLI group actions (Lemma 2.7), and

(2) a Baire-measurable homomorphism between orbit equivalence relations induces, after restricting to an invariant dense $G_{\delta}$ set, a homomorphism at the level of Becker digraphs (Proposition 2.8).

Lemma 2.7. If $Y$ is a Polish $H$-space and $H$ is a CLI group, then the Becker digraph $\mathcal{B}(Y / H)$ contains only loops.

Proof. Fix a compatible complete right-invariant metric $d$ on $H$. For a subset $A$ of $H$ we let $\operatorname{diam}(A)$ be the diameter of $A$ with respect to $d$. Let $x, y$ be elements of $Y$ with different $H$-orbits. We show that Player I has a winning strategy in $\operatorname{Emb}(x, y)$. In the $n$-th round Player $I$ plays some symmetric open neighborhood $V_{n+1}$ of the identity of $H$ with $\operatorname{diam}\left(V_{n+1}\right)<2^{-n}$ and an open neighborhood $U_{n}$ of $x$ such that the sequence $\left(U_{n}\right)$ forms a decreasing basis of neighborhoods of $x$. Let $\left(g_{n}\right)$ be the sequence of group elements chosen by Player II, and set $h_{n}:=g_{n} \cdots g_{0}$. We claim that such a sequence does not satisfy the winning condition for Player II in the Becker-embedding game. Suppose by contradiction that this is the case, and hence $\lim _{n} h_{n} y=x$. For every $n>m$ we have by right invariance of $d$ that

$$
d\left(h_{n}, h_{m}\right)=d\left(g_{n} \cdots g_{m+1}, 1\right) \leq d\left(g_{n}, 1\right)+d\left(g_{n-1}, 1\right)+\cdots+d\left(g_{m+1}, 1\right)<2^{-m} .
$$

Therefore $h_{n}$ is a $d$-Cauchy sequence with respect to $d$. Since by assumption $d$ is complete, $h_{n}$ converges to some $h \in H$. From $\lim _{n} h_{n} y=x$ and continuity of the action, we deduce that $h y=x$. This contradicts the assumption that the $H$-orbits of $x$ and $y$ are different.

Using the orbit continuity lemma (Lemma 2.5) one can then show that a Baire-measurable homomorphism preserves Becker embeddability on a comeager set. This is the content of the following proposition.

Proposition 2.8. Suppose that $G, H$ are Polish groups, $X$ is a Polish $G$-space, and $Y$ is a Polish $H$-space. Let $f: X \rightarrow Y$ be a Baire-measurable $\left(E_{G}^{X}, E_{H}^{Y}\right)$-homomorphism. Then there exists a $G$-invariant dense $G_{\delta}$ subset $X_{0}$ of $X$ such that the function $[f]: X_{0} / G \rightarrow Y / H,[x] \mapsto[f(x)]$ is a digraph homomorphism from the Becker digraph $\mathcal{B}\left(X_{0} / G\right)$ to the Becker digraph $\mathcal{B}(Y / G)$.

Proof. As in the proof of Lemma 2.5, we will use the category quantifier $\forall^{*} x \in U$ for the statement "for a comeager set of $x \in U^{\prime}$ ". Let $C$ be dense $G_{\delta}$ subsets of $X$ obtained from $f$ as in Lemma 2.5. Set $X_{0}:=$ $\left\{x \in X: \forall^{*} g \in G, g x \in C\right\}$, which is a $G$-invariant dense $G_{\delta}$ set by [12, Proposition 3.2.5 and Theorem 3.2.7]. We claim that $[f]: X_{0} / G \rightarrow Y / H,[x] \mapsto[f(x)]$ is a digraph homomorphism from the Becker $\operatorname{digraph} \mathcal{B}\left(X_{0} / G\right)$ to the Becker digraph $\mathcal{B}(Y / G)$.

Fix $x_{0}, y_{0} \in X_{0}$ such that $x_{0} \preccurlyeq \mathcal{B} y_{0}$. We want to prove that $f\left(x_{0}\right) \preccurlyeq \mathcal{B} f\left(y_{0}\right)$. Observe that $\forall^{*} g \in G$, $g x_{0} \in C \cap X_{0}$. Therefore after replacing $x_{0}$ with $g x_{0}$ for a suitable $g \in G$ we can assume that $x_{0} \in C \cap X_{0}$. Let us consider thus the Becker-embedding game $\operatorname{Emb}\left(f\left(x_{0}\right), f\left(y_{0}\right)\right)$. At the same time we consider the Beckerembedding game $\operatorname{Emb}\left(x_{0}, y_{0}\right)$ and use the fact that Player II has a winning strategy for such a game. 
In the first turn of $\operatorname{Emb}\left(f\left(x_{0}\right), f\left(y_{0}\right)\right)$, Player I plays an open neighborhood $\widehat{U}_{1}$ of $f\left(x_{0}\right)$ and an open neighborhood $\widehat{V}_{1}$ of the identity of $H$. Consider an open neighborhood $U_{1}$ of $x_{0}$ and an open neighborhood $V_{1}$ of the identity of $G$ such that for any $x \in U_{1} \cap C \cap X_{0}$ and a comeager set of $g \in V_{1}$ one has that $f(g x) \in \widehat{V}_{1} f(x)$. Consider now the round of the game $\operatorname{Emb}\left(x_{0}, y_{0}\right)$ where, in the first turn, Player I plays the neighborhood $U_{1}$ of $x_{0}$ and the neighborhood $V_{1}$ of the identity of $G$. Since by assumption Player II has a winning strategy for $\operatorname{Emb}\left(x_{0}, y_{0}\right)$, we can consider an element $g_{0}$ of $V_{1}$ which is obtained from such a winning strategy. By Remark 2.3, we can also insist that $g_{0}$ belongs to the comeager set of $g \in V_{1}$ such that $g y_{0} \in U_{1} \cap C \cap X_{0}$ and $f\left(g y_{0}\right) \in \widehat{V}_{1} f(x)$. We can then let Player II play, in the first turn of the game $\operatorname{Emb}\left(f\left(x_{0}\right), f\left(y_{0}\right)\right)$, an element $h_{0}$ of $\widehat{V}_{1}$ such that $f\left(g_{0} y_{0}\right)=h_{0} f\left(y_{0}\right)$.

At the $n$-th turn of $\operatorname{Emb}\left(f\left(x_{0}\right), f\left(y_{0}\right)\right)$, Player I plays an open neighborhood $\widehat{U}_{n}$ of $f\left(x_{0}\right)$ and an open neighborhood $\widehat{V}_{n}$ of the identity of $H$. Consider now an open neighborhood $U_{n}$ of $x_{0}$ and an open neighborhood $V_{n}$ of the identity of $G$ such that for any $x \in U_{n} \cap C \cap X_{0}$ and a comeager set of $g \in V_{n}$ one has that $f(g x) \in \widehat{V}_{n} f(x)$. Let Player I play, in the $n$-turn of $\operatorname{Emb}\left(x_{0}, y_{0}\right)$, the open neighborhoods $U_{n}$ of $x_{0}$ and $V_{n}$ of the identity of $G$. Let $g_{n-1} \in V_{n}$ be obtained from a winning strategy for Player II. By Remark 2.3 we can insist that $g_{n-1}$ belongs to the comeager set of $g \in V_{n}$ such that $g g_{n-2} \cdots g_{1} g_{0} y \in U_{n} \cap C \cap X_{0}$ and $f(g x) \in \widehat{V}_{1} f(x)$. Therefore we can let Player II play, in the $n$-th turn of the game $\operatorname{Emb}\left(f\left(x_{0}\right), f\left(y_{0}\right)\right)$, an element $h_{n-1} \in \widehat{V}_{n-1}$ such that $f\left(g_{n-1} \cdots g_{0} y\right)=h_{n-1} f\left(g_{n-1} \cdots g_{0} y\right)=h_{n-1} \cdots h_{0} y \in \widehat{U}_{n}$. Such a construction witness that Player II has a winning strategy for the game $\operatorname{Emb}\left(f\left(x_{0}\right), f\left(y_{0}\right)\right)$.

From Lemma 2.7 and Proposition 2.8 one can immediately deduce the following criterion to show that the orbit equivalence relation of a Polish group action is not Borel reducible to the orbit equivalence relation of CLI group action.

Theorem 2.9. Suppose that $X$ is a Polish $G$-space. If for any $G$-invariant dense $G_{\delta}$ subset $C$ of $X$ there exist $x, y \in C$ with different $G$-orbits such that $x \preccurlyeq \mathcal{B} y$, then for any $G$-invariant dense $G_{\delta}$ subset $C$ of $X$ the relation $E_{C}^{X}$ is not CLI-classifiable.

Proof. Suppose that $H$ is a CLI group, and $Y$ is a Polish $H$-space. Suppose that $D$ is a $G$-invariant dense $G_{\delta}$ subset of $X$, and $f: D \rightarrow Y$ is a Borel $\left(E_{G}^{D}, E_{H}^{Y}\right)$-homomorphism. Then by Proposition 2.8 there exists a $G$-invariant dense $G_{\delta}$ subset $C$ of $D$ such that $[f]: C / G \rightarrow Y / H$ is a digraph homomorphisms for the Becker digraphs $\mathcal{B}(C / G)$ and $\mathcal{B}(Y / H)$. By assumption there exist elements $x, y$ of $C$ with different $G$-orbits such that $x \preccurlyeq \mathcal{B} y$. Therefore $f(x) \preccurlyeq \mathcal{B} f(y)$. Since $H$ is CLI we have by Lemma 2.7 that $f(x)$ and $f(y)$ belong to the same $H$-orbit. Therefore $f$ is not a reduction from $E_{G}^{D}$ to $E_{H}^{Y}$.

2.4. Applications. Suppose that $E$ is an equivalence relation on a Polish space $X$. Recall that the FriedmanStanley jump $E^{+}$of $E$ [12, Definition 8.3.1] - see also [10] - is the equivalence relation on the standard Borel space $X^{\mathbb{N}}$ of sequences of elements of $X$ defined by $\left(x_{n}\right) E^{+}\left(y_{n}\right)$ if and only if $\left\{\left[x_{n}\right]_{E}: n \in \mathbb{N}\right\}=\left\{\left[y_{n}\right]_{E}: n \in \mathbb{N}\right\}$.

In particular one can start with the relation $=$ of equality on a perfect Polish space $X$. The corresponding Friedman-Stanley jump is the relation $=^{+}$on $X^{\omega}$ defined by $\left(x_{n}\right)={ }^{+}\left(y_{n}\right)$ if and only if the sequences $\left(x_{n}\right)$ and $\left(y_{n}\right)$ have the same range. With respect to Borel reducibility, $={ }^{+}$is the most complicated (essentially) $\boldsymbol{\Pi}_{3}^{0}$ equivalence relation [12, Theorem 12.5.5]; see also [17].

Hjorth has proven in [15, Theorem 5.19] that $={ }^{+}$is not Borel reducible to the orbit equivalence relation of a continuous action of an abelian Polish group. As remarked in [15, page 663], Hjorth's proof uses a metamathematical argument involving forcing and Stern's absoluteness principle. Similar methods are used in [20, Theorem 17.1.3] to prove that $={ }^{+}$is not Borel reducible to the orbit equivalence relation of a Borel action of a CLI group. This is obtained as a consequence of a general result concerning pinned equivalence relations; see [20, Definition 17.1.2]. To our knowledge, the argument below provides the first entirely classical proof of this result.

Let $\sigma: X^{\mathbb{N}} \rightarrow X^{\mathbb{N}}$ be the unilateral shift $\left(x_{1}, x_{2}, \ldots\right) \mapsto\left(x_{2}, x_{3}, \ldots\right)$. We consider the restriction of $=^{+}$to the dense $G_{\delta}$ subset $Y$ of $X^{\mathbb{N}}$ that consists of injective sequences. Observe that this is the orbit equivalence relation of the canonical action of $S_{\infty}$ on $X^{\mathbb{N}}$ obtained by permuting the indices.

Theorem 2.10. Let $Z \subset Y$ be a nonempty $S_{\infty}$-invariant $G_{\delta}$ set such that $\sigma[Z]=Z$. The restriction of $=^{+}$to any $S_{\infty}$-invariant dense $G_{\delta}$ subset of $Z$ is not Borel reducible to a Borel action of a CLI group on a standard Borel space.

Proof. Let $E$ be the restriction of $=^{+}$to $Z$. As observed before, $E$ is the orbit equivalence relation of the canonical action $S_{\infty} \curvearrowright Z \subset Y \subset X^{\mathbb{N}}$ given by permuting the coordinates. We apply Proposition 2.9. Let $C$ be an $S_{\infty}$-invariant dense $G_{\delta}$ subset of $Z$. We need to prove that there exist $x, y \in C$ with different orbits such 
that $x \preccurlyeq \mathcal{B} y$. For $x=\left(x_{n}\right) \in Y$ we let $\operatorname{Ran}(x)$ be the set $\left\{x_{n}: n \in \mathbb{N}\right\}$. It is not difficult to see directly that, for $x, y \in Y, x \preccurlyeq \mathcal{B} y$ if and only if $\operatorname{Ran}(x) \subset \operatorname{Ran}(y)$. When $X$ is the Cantor space $2^{\mathbb{N}}$, this assertion is a particular instance of [3, Proposition 3.D.2]. Indeed, in this case $X^{\mathbb{N}}$ can be seen as the space $\operatorname{Mod}(\mathcal{L})$ of $\mathcal{L}$-structures endowed with the topology $t_{F}$, where $\mathcal{L}$ is the language containing a countably infinite collection of unitary relations and $F$ is the fragment consisting of atomic formulas. Observe that $\sigma: Z \rightarrow Z$ is continuous, open, and surjective. Therefore, since $C$ is a dense $G_{\delta}$ subset of $Z$, we have that there exists a comeager subset $C_{0}$ of $C$ such that, for every $x \in C_{0}, \sigma^{-1}(x) \cap C$ is a comeager subset of $\sigma^{-1}(x)$; see [31, Theorem A.1]. Pick now $x \in C_{0}$ and $y \in \sigma^{-1}(x) \cap C$. It is clear that $x \preccurlyeq \mathcal{B} y$ and $x, y$ lie in different $S_{\infty}$-orbits. This concludes the proof.

We now apply Theorem 2.10 to obtain information about the orbit equivalence relation of some canonical actions of the uniteray group $\mathcal{U}(\mathcal{H})$. Let $\mathcal{H}$ be the separable infinite-dimensional Hilbert space, and let $\mathcal{U}(\mathcal{H})$ be the group of unitary operators on $\mathcal{H}$. This is a Polish group when endowed with the weak operator topology; see [5, Proposition I.3.2.9]. The group $\mathcal{U}(\mathcal{H})$ admits a canonical action by conjugation on itself and on the space $\mathcal{B}(\mathcal{H})_{s a}$ of selfadjoint operators.

Theorem 2.11. The following relations are not Borel reducible to a Borel action of a CLI group on a standard Borel space:

(1) unitary equivalence of unitary operators;

(2) unitary equivalence of selfadjoint operators.

Proof. As in Theorem 2.10 we consider the equivalence relation $=^{+}$on the set $X^{\mathbb{N}}$ of sequences of elements of a perfect Polish space $X$. Fix an orthonormal basis $\left(e_{n}\right)$ of $\mathcal{H}$. Let $X$ be the circle group $\mathbb{T}$, and $Y \subset \mathbb{T}^{\mathbb{N}}$ be the set of injective sequences. The map $f: Y \rightarrow \mathcal{U}(\mathcal{H})$ which sends an element $\left(\lambda_{n}\right) \in Y$ to the unitary operator

$$
\left(e_{n}\right) \mapsto\left(\lambda_{n} e_{n}\right)
$$

is a Borel reduction from $=\left.{ }^{+}\right|_{Y}$ to unitary equivalence of unitary operators. The proof of selfadjoint operators is the same, where one replaces $\mathbb{T}$ with $[0,1]$.

\section{A game-Theoretic APPRoACh to turbulence}

3.1. Hjorth's turbulence theory. Suppose that $\mathcal{L}=\left(R_{i}\right)_{i \in I}$ is a countable first order relational language, where $R_{i}$ is a relation symbol with arity $n_{i}$. We denote as above by $\operatorname{Mod}(\mathcal{L})$ the Polish $S_{\infty}$-space of $\mathcal{L}$-structures with support $\mathbb{N}$. Recall that a Polish group $G$ is called non-Archimedean if it admits a neighborhood basis of the identity of open subgroups or, equivalently, it is isomorphic to a closed subgroup of $S_{\infty}$; see [4, Theorem 1.5.1]. A relation $E$ is classifiable by countable structures if it is Borel reducible to the isomorphism relation in $\operatorname{Mod}(\mathcal{L})$ for some countable first order relational language $\mathcal{L}$. This is equivalent to the assertion that $E$ is Borel reducible to the orbit equivalence relation of a Borel action of a non-Archimedean Polish group $G$ on a standard Borel space by [4, Theorem 5.1.11] and [12, Theorem 3.5.2, Theorem 11.3.8].

Turbulence is a dynamical condition on a Polish $G$-space $X$ which is an obstruction of classifiability of $E_{G}^{X}$ by countable structures. We now recall here the fundamental notions of the theory of turbulence, developed by Hjorth in [16]. Suppose that $X$ is a Polish $G$-space, $x \in X, U$ is a neighborhood of $x$, and $V$ is a neighborhood of the identity in $G$. The local orbit $\mathcal{O}(x, U, V)$ is the smallest subset of $U$ with the property that $x \in \mathcal{O}(x, U, V)$, and if $g \in V, x \in \mathcal{O}(x, U, V)$, and $g x \in U$, then $g x \in \mathcal{O}(x, U, V)$. A point $x \in X$ is called turbulent if it has dense orbit and, for any neighborhood $U$ of $x$ and neighborhood $V$ of the identity in $G$, the closure of $\mathcal{O}(x, U, V)$ is a neighborhood of $x$. A Polish $G$-space $X$ is preturbulent if every point $x \in X$ is turbulent, and turbulent if every point $x \in X$ is turbulent and has meager orbit.

An equivalence relation $E$ on a Polish space $X$ is generically $S_{\infty}$-ergodic if, for any Polish $S_{\infty}$-space $Y$ and Baire-measurable $\left(E, E_{S_{\infty}}^{Y}\right)$-homomorphism, there exists a comeager subset of $X$ that is mapped by $f$ to a single $S_{\infty}$-orbit. By [12, Theorem 3.5.2, Theorem 11.3.8], this is equivalent to the assertion that, for any nonArchimedean Polish group $H$, Polish $H$-space $Y$, and Baire measurable $\left(E, E_{H}^{Y}\right)$-homomorphisms, there exists a comeager subset of $X$ that is mapped by $f$ to a single $H$-orbit. The following is the main result in Hjorth's turbulence theory, providing a dichotomy for preturbulent Polish $G$-spaces.

Theorem 3.1 (Hjorth). Suppose that $X$ is a preturbulent Polish G-space. Then the associated orbit equivalence relation $E_{G}^{X}$ is generically $S_{\infty}$-ergodic. In particular, either $X$ has a dense $G_{\delta}$ orbit, or the restriction of $E_{G}^{X}$ to any comeager subset of $X$ is not classifiable by countable structure. 
In this section, for each Polish $G$-space $X$, we define a graph structure $\mathcal{H}(X / G)$ with domain the quotient $X / G=\{[x]: x \in X\}$ of $X$ via the action of $G$. We call this the Hjorth graph associated with the $G$-space $X$. An (induced) subgraph of $\mathcal{H}(X / G)$ is of the form $\mathcal{H}(C / G)$, where $C$ is an invariant subset of $X$. We view Hjorth's turbulence theorem as a corollary of the following facts:

(1) $\mathcal{H}(X / G)$ contains only loops if $G$ is non-Archimedean;

(2) $\mathcal{H}(X / G)$ is a clique if the action of $G$ on $X$ is preturbulent;

(3) given a Polish $G$-space $X$ and a Polish $H$-space $Y$, a Baire measurable $\left(E_{G}^{X}, E_{H}^{Y}\right)$-homomorphism $f$ induces, after restricting to an invariant dense $G_{\delta}$ set, a graph homomorphism between the corresponding Hjorth graphs.

3.2. The Hjorth-isomorphism game. We start by defining a game associated with points of a given Polish $G$-space, which captures isomorphism in the case of Polish $S_{\infty}$-spaces.

Definition 3.2. Suppose that $X$ is a Polish $G$-space, and $x, y \in X$. We consider the Hjorth-isomorphism game Iso $(x, y)$ played between two players as follows. Set $x_{0}:=x, y_{0}:=y, U_{0}^{y}:=X$, and $V_{0}^{y}=G$.

(1) In the first turn, Player I plays an open neighborhood $U_{0}^{x}$ of $x_{0}$ and an open neighborhood $V_{0}^{x}$ of the identity in $G$. Player II replies with an element $g_{0}^{y}$ in $G$.

(2) In the second turn, Player I then plays an open neighborhood $U_{1}^{y}$ of $y_{1}:=g_{0}^{y} y_{0}$ and an open neighborhood $V_{1}^{y}$ of the identity of $G$, and Player II replies with an element $g_{0}^{x}$ in $G$.

$(2 \mathrm{n}+1)$ At the $(2 n+1)$-st turn, Player I plays an open neighborhood $U_{n}^{x}$ of $x_{n}:=g_{n-1}^{x} x_{n-1}$ and an open neighborhood $V_{n}^{x}$ of the identity of $G$, and Player II responds with an element $g_{n}^{y}$ of $G$.

$(2 \mathrm{n}+2)$ At the $(2 n+2)$-nd turn, Player I plays an open neighborhood $U_{n+1}^{y}$ of $y_{n+1}:=g_{n}^{y} y_{n}$ and an open neighborhood $V_{n+1}^{y}$ of the identity of $G$, and Player II responds with an element $g_{n}^{x}$ of $G$.

The game proceed in this way, producing sequences $\left(x_{n}\right)$ and $\left(y_{n}\right)$ of elements of $X$, sequences $\left(g_{n}^{x}\right)$ and $\left(g_{n}^{y}\right)$ of elements of $G$, sequences $\left(U_{n}^{x}\right)$ and $\left(U_{n}^{y}\right)$ of open subsets of $X$, and sequences $\left(V_{n}^{x}\right)$ and $\left(V_{n}^{y}\right)$ of open neighborhoods of the identity in $G$. Player II wins the game if, for every $n \geq 0$,

- $y_{n+1} \in U_{n}^{x}$ and $x_{n} \in U_{n}^{y}$,

- $g_{n}^{y}=h_{k} \cdots h_{0}$ for some $k \geq 0$ and $h_{0}, \ldots, h_{k} \in V_{n}^{y}$ such that $h_{i} \cdots h_{0} y_{n} \in U_{n}^{y}$ for $i \leq k$,

- $g_{n}^{x}=h_{k} \cdots h_{0}$ for some $k \geq 0$ and $h_{0}, \ldots, h_{k} \in V_{n}^{x}$ such that $h_{i} \cdots h_{0} x_{n} \in U_{n}^{x}$ for $i \leq k$.

We write $x \sim_{\mathcal{H}} y$ and we say that $x, y$ are Hjorth-isomorphic if Player II has a winning strategy for the Hjorth game $\mathcal{H}(x, y)$.

Remark 3.3. As in the case of the Becker-embedding game - see Remark 2.3 - it is not difficult to see that, if Player II has a winning strategy for the Hjorth game as described above, then it also has a winning strategy for the same game with the additional winning conditions that $g_{n}^{x}=h_{k} \cdots h_{0}$ for some $h_{0}, \ldots, h_{k}$ from a given comeager subset of $V_{n}^{x}$ such that $h_{i} \cdots h_{0} x_{n}$ belongs to a given comeager subset $X_{0}$ of $X$ for $i=0, \ldots, k$, provided that the set of $h \in G$ such that $h x \in X_{0}$ is comeager. Similarly one can add the winning conditions that $g_{n}^{y}=h_{k} \cdots h_{0}$ for some $h_{0}, \ldots, h_{k}$ from a given comeager subset of $V_{n}^{y}$ such that $h_{i} \cdots h_{0} y_{n}$ belongs to a given comeager subset $X_{0}$ of $X$, provided that the set of $h \in G$ such that $h y \in X_{0}$ is comeager.

The relation $\sim_{\mathcal{H}}$ is an equivalence relation on $X$ which we call Hjorth isomorphism. It is clear that Hjorth isomorphism is a coarsening of the orbit equivalence relation $E_{G}$ on $G$. Furthermore if $x \sim \mathcal{H} y, x^{\prime}$ belongs to the $G$-orbit of $x$, and $y^{\prime}$ belongs to the $G$-orbit of $y$, then $x^{\prime} \sim_{\mathcal{H}} y^{\prime}$. Let as before $X / G$ be the space of $G$-orbits of elements of $X$. The Hjorth-graph $\mathcal{H}(X / G)$ associated with the Polish $G$-space $X$ is symmetric, reflexive graph on $X / G$ given by declaring that there exists an edge between the orbit $[x]$ of $x$ and the orbit $[y]$ of $y$ if and only if $x \sim \mathcal{H} y$. We call $\mathcal{H}(X / G)$ the Hjorth graph associated with the Polish $G$-space $X$. One can similarly define the Hjorth graph $\mathcal{H}(C / G)$ for any invariant subset $C$ of $X$. A comeager subgraph $\mathcal{G}$ of $\mathcal{H}(X / G)$ is a graph of the form $\mathcal{H}(C / G)$, for some invariant comeager subset $C$ of $X$.

3.3. Generic homomorphisms between Hjorth graphs. We now proceed to the proof of the properties of Hjorth graphs stated at the end of Subsection 3.1. In the following, for a subset $V$ of $G$ and $k \in \mathbb{N}$ let $V^{k}$ be the set of elements of $G$ that can be written as the product of $k$ elements from $V$.

Lemma 3.4. Suppose that $H$ is a non-Archimedean Polish group, and $Y$ is a Polish $H$-space. Then the Hjorth graph $\mathcal{H}(Y / H)$ contains only loops.

Proof. Suppose that $G$ is a non-Archimedean Polish group. Fix a compatible complete metric $d$ on $X$, and a compatible complete metric $d_{G}$ on $G$. We denote by $\operatorname{diam}(A)$ the diameter of a subset $A$ of $X$ with respect 
to the metric $d$, and by $\operatorname{cl}(A)$ the closure of $A$. Suppose that Player II has a winning strategy for the Hjorthisomorphism game Iso $(x, y)$. We want to show that $x$ and $y$ belong to the same orbit. This can be seen by letting Player I play open subsets $U_{n}^{x}$ and $U_{n}^{y}$ of $X$ such that $\operatorname{cl}\left(U_{n+1}^{y}\right) \subset U_{n}^{x}, \operatorname{cl}\left(U_{n}^{x}\right) \subset U_{n}^{y}, \operatorname{diam}\left(U_{n}^{x}\right) \leq 2^{-n}$, $\operatorname{diam}\left(U_{n+1}^{y}\right) \leq 2^{-n}$, and open subgroups $V_{n}^{x}$ and $V_{n}^{y}$ of $G$ such that

$$
\begin{aligned}
& V_{n}^{x} \subset\left\{g \in G: d_{G}\left(g g_{n-1}^{x} \cdots g_{0}^{x}, g_{n-1}^{x} \cdots g_{0}^{x}\right)<2^{-n}\right\} \\
& V_{n}^{y} \subset\left\{g \in G: d_{G}\left(g g_{n-1}^{y} \cdots g_{0}^{y}, g_{n-1}^{y} \cdots g_{0}^{y}\right)<2^{-n}\right\} .
\end{aligned}
$$

Let then $\left(x_{n}\right)$ and $\left(y_{n}\right)$ be the sequences of elements of $X$ and $\left(g_{n}^{x}\right)$ and $\left(g_{n}^{y}\right)$ be the sequences of elements of $G$ obtained from the corresponding round of the Hjorth game. Then the assumptions on $U_{n}^{x}$ and $U_{n}^{y}$ guarantee that the sequences $\left(x_{n}\right)$ and $\left(y_{n}\right)$ converge to the same point $z$ of $X$. The assumptions on $V_{n}^{x}$ and $V_{n}^{y}$ guarantee that the sequences $\left(g_{n}^{x} g_{n-1}^{x} \cdots g_{0}^{x}\right)_{n \in \omega}$ and $\left(g_{n}^{y} g_{n-1}^{y} \cdots g_{0}^{y}\right)_{n \in \omega}$ converge in $H$ to elements $g_{\infty}^{x}$ and $g_{\infty}^{y}$ such that $g_{\infty}^{x} x=z$ and $g_{\infty}^{y} y=z$. This shows that $x$ and $y$ belong to the same orbit.

Lemma 3.5. Suppose that $X$ is a preturbulent Polish $G$-space. Then the Hjorth graph $\mathcal{H}(X / G)$ is a clique.

Proof. Suppose that $X$ is a preturbulent Polish $G$-space. Fix $x, y \in X$. We want to prove that Player II has a winning strategy for the Hjorth game $\mathcal{H}(x, y)$. We begin with a preliminary observation. Suppose that $z \in X$, $U$ is an open neighborhood of $z$, and $V$ is an open neighborhood of the identity in $G$. Let $\mathcal{I}(z, U, V)$ be the interior of the closure of the local orbit $\mathcal{O}(z, U, V)$. Since $z$ is turbulent, $\mathcal{I}(z, U, V)$ contains $z$. It is not difficult to see that, for any $w \in \mathcal{I}(z, U, V)$, the local orbit $\mathcal{O}(w, \mathcal{I}(z, U, V), V)$ is dense in $\mathcal{I}(z, U, V)$. We use this observation to conclude that Player II has a winning strategy, which we proceed to define. As in the definition of the Hjorth game, we let $x_{0}=x, y_{0}=y, U_{0}^{y}=X$, and $V_{0}^{y}=G$. At the $(2 n+1)$-st turn Player II plays an element $g_{n}^{y}=h_{k} \cdots h_{0} \in\left(V_{n}^{y}\right)^{k}$ for some $k \geq 1$ such that $y_{n+1}=g_{n}^{y} y_{n} \in \mathcal{I}\left(x_{n}, U_{n}^{x}, V_{n}^{x}\right)$ and $h_{i} \cdots h_{0} y_{n} \in U_{n}^{y}$ for $i \leq k$, while at the $(2 n+2)$-nd turn Player II plays an element $g_{n}^{x}=h_{k} \cdots h_{0} \in\left(V_{n}^{x}\right)^{k}$ for some $k \geq 1$ such that $x_{n+1}=g_{n}^{x} x_{n} \in \mathcal{I}\left(y_{n+1}, U_{n+1}^{y}, V_{n+1}^{y}\right)$ and $h_{i} \cdots h_{0} x_{n} \in U_{n}^{x}$ for $i \leq k$. Such a choice is possible at the 1-st turn since $y$ has dense orbit. It is possible at the $(2 n+2)$-nd turn $(n \geq 0)$ since $y_{n+1} \in \mathcal{I}\left(x_{n}, U_{n}^{x}, V_{n}^{x}\right)$ and for every $w \in \mathcal{I}\left(x_{n}, U_{n}^{x}, V_{n}^{x}\right)$ the local orbit $\mathcal{O}\left(w, \mathcal{I}\left(x_{n}, U_{n}^{x}, V_{n}^{x}\right), V_{n}^{x}\right)$ is dense in $\mathcal{I}\left(x_{n}, U_{n}^{x}, V_{n}^{x}\right)$. It is possible at the $(2 n+1)$-st turn $(n \geq 1)$ since $x_{n} \in \mathcal{I}\left(y_{n}, U_{n}^{y}, V_{n}^{y}\right)$ and for any $w \in \mathcal{I}\left(y_{n}, U_{n}^{y}, V_{n}^{y}\right)$ the local orbit $\mathcal{O}\left(w, \mathcal{I}\left(y_{n}, U_{n}^{y}, V_{n}^{y}\right), V_{n}^{y}\right)$ is dense in $\mathcal{I}\left(y_{n}, U_{n}^{y}, V_{n}^{y}\right)$. This concludes the proof that Player II has a winning strategy for the Hjorth game $\mathcal{H}(x, y)$.

Proposition 3.6. Suppose that $G, H$ are Polish groups, $X$ is a Polish $G$-space, and $Y$ is a Polish $H$-space. If $f$ is a Baire-measurable $\left(E_{G}^{X}, E_{H}^{Y}\right)$-homomorphism, then there exists a $G$-invariant dense $G_{\delta}$ subset $X_{0}$ of $X$ such that the function $X_{0} / G \rightarrow Y / H,[x] \mapsto[f(x)]$ is a homomorphism from the Hjorth graph $\mathcal{H}\left(X_{0} / G\right)$ to the Hjorth graph $\mathcal{H}(Y / H)$.

Proof. We proceed as in the proof of Proposition 2.8. Let $C$ be dense $G_{\delta}$ subsets of $X$ obtained from $f$ as in Lemma 2.5. Set $X_{0}:=\left\{x \in X: \forall^{*} g \in G, g x \in C\right\}$, which is a $G$-invariant dense $G_{\delta}$ set by [12, Proposition 3.2 .5 and Theorem 3.2.7]. We claim that $X_{0} / G \rightarrow Y / H,[x] \mapsto[f(x)]$ is a graph homomorphism from the Hjorth graph $\mathcal{H}\left(X_{0} / G\right)$ to the Hjorth graph $\mathcal{H}(Y / H)$.

Fix $x_{0}, y_{0} \in X_{0}$ such that $x_{0} \sim_{\mathcal{H}} y_{0}$. We want to prove that $f\left(x_{0}\right) \sim_{\mathcal{H}} f\left(y_{0}\right)$. Observe that $\forall^{*} g \in G$, $g x_{0} \in C \cap X_{0}$. Therefore after replacing $x_{0}$ with $g x_{0}$ for a suitable $g \in G$ we can assume that $x_{0} \in C \cap X_{0}$. In this case one can define, similarly as in the proof of Proposition 2.8, a winning strategy for Player II for Iso $\left(f\left(x_{0}\right), f\left(y_{0}\right)\right)$ from a winning strategy for Player II for Iso $\left(x_{0}, y_{0}\right)$ using Remark 3.3 and the choice of $C$.

It is now easy to see that Theorem 3.1 is an immediate consequence of Lemma 3.4 and Lemma 3.5 together with Proposition 3.6.

\section{Groupoids}

4.1. Polish groupoids. The goal of this section is to observe that the proofs above apply equally well in the setting of Polish groupoids as introduced in $[28,33,34]$. A groupoid $G$ is a small category where every morphism (also called arrow) is invertible. By identifying any object with the corresponding identity arrow, one can regard the set $G^{0}$ of objects of $G$ as a subset of $G$. The source and range maps $s, r: G \rightarrow G^{0}$ assign to every arrow in $G$ its domain (or source) and codomain (or range). The set $G^{2}$ of composable arrows is the set of pairs $(\gamma, \rho)$ of arrows from $G$ such that $s(\gamma)=r(\rho)$. Composition of arrows is a function $G^{2} \rightarrow G,(\gamma, \rho) \rightarrow \gamma \rho$. If $A, B \subset G$, then we denote by $A B$ the set $\left\{\gamma \rho:(\gamma, \rho) \in G^{2} \cap(A \times B)\right\}$. If $x \in G^{0}$ and $A \subset G$, then we let $A x:=A\{x\}=\{\gamma \in G: s(\gamma)=x\}$ and $x A:=\{x\} A=\{\gamma \in G: r(\gamma)=x\}$.

A Polish groupoid is a groupoid $G$ endowed with a topology such that 
(1) there exists a countable basis $\mathcal{B}$ of Polish open sets,

(2) composition and inversion of arrows are continuous and open,

(3) the sets $G x$ and $x G$ are Polish subspaces for every $x \in G^{0}$, and

(4) the set of objects $G^{0}$ is a Polish subspace.

A Polish groupoid is not required to be globally Hausdorff. Many Polish groupoids arising in the applications, such as the locally compact groupoids associated with foliations of manifolds, are not Hausdorff; see [32, Chapter 2].

Suppose that $H$ is a Polish group. One can associate with any Polish $H$-space $X$ a Polish groupoid $H \ltimes X-$ the action groupoid - that completely encodes the action. Such a groupoid has the Cartesian product $H \times X$ as set of arrows (endowed with the product topology), and $\left\{\left(1_{H}, x\right): x \in X\right\}$ as set of objects. Source and range maps are defined by $s(h, x)=\left(1_{H}, x\right)$ and $r(h, x)=\left(1_{H}, h x\right)$. Composition is given by $(h, x)\left(h^{\prime}, y\right)=\left(h h^{\prime}, y\right)$ whenever $x=h^{\prime} y$. In this way one can regard continuous actions of Polish groups on Polish spaces as a particular instance of Polish groupoids. One can also consider continuous actions of Polish groupoids on Polish spaces, but these can be in turn regarded as Polish groupoids via a similar construction as the one described above. The class of Polish groupoids is also closed under taking restrictions. If $X$ is a $G_{\delta}$ subset of the set of objects of a Polish groupoid $G$, then the restriction $\left.G\right|_{X}$ is the collection of arrows of $G$ with source and range in $X$, endowed with the induced Polish groupoid structure. More information about Polish groupoids can be found in [28].

Given a Polish groupoid $G$, the orbit equivalence relation $E_{G}$ is the equivalence relation on $G^{0}$ defined by setting $x E_{G} y$ if and only if $x, y$ are source and range of an arrow from $G$. The orbit of an object in $G$ is the $E_{G}$-class of $x$.

4.2. Turbulence for Polish groupoids. The notion of (pre)turbulence for Polish groupoid has been considered in [13, Section 4]. Suppose that $G$ is a Polish groupoid, $x$ is an object of $G$, and $U$ is a neighborhood of $x$ in $G$. The local orbit $\mathcal{O}(x, U)$ is the smallest subset of $U \cap G^{0}$ with the property that $x \in \mathcal{O}(x, U)$, and if $\gamma \in U$ is such that $s(\gamma) \in \mathcal{O}(x, U)$, then $r(\gamma) \in \mathcal{O}(x, U)$. An object $x$ is called turbulent if it has orbit dense in $G^{0}$ and, for any neighborhood $U$ of $x$, the closure of $\mathcal{O}(x, U)$ is a neighborhood of $x$ in $G^{0}$. A Polish groupoid is preturbulent if every object is turbulent, and turbulent if every object is turbulent and has orbit meager in $G^{0}$. It is not difficult to see that these definitions are consistent with the ones for Polish group actions, when a Polish group action is identified with its associated action groupoid.

Suppose that $G$ is a Polish groupoid, and $x, y \in G^{0}$ are two objects of $G$. The Hjorth-isomorphism game Iso $(x, y)$ can be defined similarly as in Definition 3.2. Set $x_{0}:=x, y_{0}:=y, U_{0}^{y}=G$, and $V_{0}^{y}=G$. In this case, in the first turn Player I plays an open neighborhood $U_{0}^{x}$ of $x_{0}$ in $G$ and Player II replies with an element $\gamma_{0}^{y}$ of $G$ with $s\left(\gamma_{0}^{y}\right)=y_{0}$. In the second turn, Player I plays an open neighborhood $U_{1}^{y}$ of $y_{1}:=r\left(\gamma_{0}^{y}\right)$ in $G$ and an element $\gamma_{0}^{x}$ of $G$ with $s\left(\gamma_{0}^{x}\right)=x_{0}$. At the $(2 n+1)$-st turn, Player I plays an open neighborhood $U_{n}^{x}$ of $x_{n}:=r\left(\gamma_{n-1}^{x}\right)$ in $G$, and Player II responds with an element $\gamma_{n}^{y}$ of $G$ with $s\left(\gamma_{n}^{y}\right)=y_{n}$. At the $(2 n+2)$-nd turn, Player I plays an open neighborhood $U_{n+1}^{y}$ of $y_{n+1}:=r\left(\gamma_{n}^{y}\right)$ in $G$, and Player II responds with an element $\gamma_{n}^{x}$ of $G$.

The game then produces sequences $\left(x_{n}\right),\left(y_{n}\right)$ of objects of $G$, sequences $\left(\gamma_{n}^{x}\right),\left(\gamma_{n}^{y}\right)$ of arrows in $G$, and sequences $\left(U_{n}^{x}\right),\left(U_{n}^{y}\right)$ of open subsets of $G$. Player II wins the game if, for every $n \geq 0$,

- $y_{n+1} \in U_{n}^{x}$ and $x_{n} \in U_{n}^{y}$,

- $\gamma_{n}^{y}=\rho_{1}^{y} \rho_{2}^{y} \cdots \rho_{k}^{y}$ for some $k \geq 1$ and $\rho_{i}^{y} \in V_{n}^{x}$ for $i=1,2, \ldots, k$, and $\gamma_{n}^{x}=\rho_{1}^{x} \cdots \rho_{k}^{x}$ for some $k \geq 1$ and $\rho_{i}^{x} \in V_{n}^{y}$ for $i=1,2, \ldots, k$.

As in the case of Polish group actions, this defines an equivalence relation $\sim_{\mathcal{H}}$ (Hjorth-isomorphism) on the set of objects of $G$, by letting $x \sim_{\mathcal{H}} y$ whenever Player II has a winning strategy for the Hjorth-isomorphism game Iso $(x, y)$. Adding to the winning conditions in the Hjorth-isomorphism game the requirement that $r\left(\gamma_{n}^{x}\right)$ belongs to a given comeager subset $X$ of $G^{0}$ and that $\gamma_{n}^{x}$ belongs to a given comeager subset of $G x_{n}$ yields an equivalent game, provided that the set of $\gamma \in G x$ such that $r(\gamma) \in X$ is comeager. The same applies to $y$. The Hjorth-isomorphism relation on $G^{0}$ defines a graph structure $\mathcal{H}(G)$ on the space of $G$-orbits, which we call the Hjorth graph of $G$. The same proof as Lemma 3.5 shows that if $G$ is a preturbulent Polish groupoid, then the Hjorth graph $\mathcal{H}(G)$ is a clique. The analogue of Lemma 2.5 for Polish groupoids has been proved in [13, Lemma 4.5]. Using this one can then prove the analog of Proposition 3.6 and deduce the following result.

Theorem 4.1. Suppose that $G$ is a preturbulent Polish groupoid. Then the associated orbit equivalence relation $E_{G}$ is generically $S_{\infty}$-ergodic. 
Theorem 4.1 recovers [13, Theorem 4.3], and can be seen as the groupoid version of Theorem 3.1 for Polish groupoids.

Since the operations in the groupoid $G$ are continuous and open, one can reformulate the Hjorth-isomorphism game Iso $(x, y)$ as presented above by letting Player II play open sets rather than groupoid elements. Fix a countable basis $\mathcal{B}$ of Polish open subsets of $G$. In this formulation of the game, Player I plays elements $U_{n}^{x}, U_{n+1}^{y}$ of $\mathcal{B}$ for $n \geq 0$ and player II plays elements $W_{n}^{x}, W_{n}^{y}$ of $\mathcal{B}$ for $n \geq 0$. The winning conditions are then, setting $U_{0}^{y}=G$,

- $r\left[W_{n+1}^{y}\right] \subset U_{n}^{x}$ and $r\left[W_{n}^{x}\right] \subset U_{n}^{y}$,

- $W_{n}^{y} \subset\left(U_{n}^{y}\right)^{k}$ for some $k \geq 1$ and $W_{n}^{x} \subset\left(U_{n}^{y}\right)^{k}$ for some $k \geq 1$,

- $y \in s\left[W_{n}^{y} \cdots W_{0}^{y}\right]$ and $x \in s\left[W_{n}^{x} \cdots W_{0}^{x}\right]$.

Such a version of the Hjorth-isomorphism game fits in the framework of Borel games as described in [22, Section 2.A]. In fact, this is an open game for Player I and closed for Player II, which allows one to define an $\omega_{1}$-valued rank for strategies of Player I [22, Exercise 20.2]. Insisting that Player I only has winning strategies of rank at least $\alpha \in \omega_{1}$ (or no winning strategy at all) gives a hierarchy of equivalence relations $\sim_{\alpha}$ indexed by countable ordinals, whose intersection is the Hjorth isomorphism relation.

4.3. Becker-embeddings for Polish groupoids. Similarly as for the Hjorth-isomorphism game, the Beckerembedding game $\operatorname{Emb}(x, y)$ can be defined whenever $x, y$ are objects in a Polish groupoid $G$. This gives a notion of Becker embedding for objects $G$, by letting $x \preccurlyeq \mathcal{B} y$ if and only if Player II has a winning strategy for $\operatorname{Emb}(x, y)$. In turn this induces a digraph structure $\mathcal{B}(G)$ on the space of $G$-orbits.

One can prove the groupoid analog of Proposition 2.8 in a similar fashion, by replacing Lemma 2.5 with [13, Lemma 4.5]. One can then deduce the following generalization of Theorem 2.9 to Polish groupoids.

Theorem 4.2. Suppose that $G$ is a Polish groupoid. If for any invariant dense $G_{\delta}$ subset $C$ of $G^{0}$ there exist $x, y \in C$ with different orbits such that $x \preccurlyeq \mathcal{B} y$, then the orbit equivalence relation $E_{G}$ is not CLI-classifiable.

As for the case of the Hjorth-isomorphism game, one can also describe the Becker-embedding game Emb $(x, y)$ for objects $x, y$ in a Polish groupoid $G$ as an open game for Player I and closed for Player II. This allows one to define an $\omega_{1}$-valued rank for strategies for Player I. Again, insisting that Player I only has winning stategies of rank at least $\alpha \in \omega_{1}$ gives a hierarchy or preorder relations $\preccurlyeq_{\alpha}$ indexed by countable ordinals, whose intersection is the Becker-embeddability preorder.

\section{REFERENCES}

1. Hiroshi Ando and Yasumichi Matsuzawa, On Borel equivalence relations related to self-adjoint operators, Journal of Operator Theory 74 (2015), no. 1, 183-194.

2. $\longrightarrow$, The Weyl-von Neumann theorem and Borel complexity of unitary equivalence modulo compacts of self-adjoint operators, Proceedings of the Royal Society of Edinburgh, Section A 145 (2015), no. 06, 1115-1144.

3. Howard Becker, Polish group actions: Dichotomies and generalized elementary embeddings, Journal of the American Mathematical Society 11 (1998), no. 2, 397-449.

4. Howard Becker and Alexander S. Kechris, The descriptive set theory of Polish group actions, London Mathematical Society Lecture Note Series, vol. 232, Cambridge University Press, Cambridge, 1996.

5. Bruce Blackadar, Operator algebras, Encyclopaedia of Mathematical Sciences, vol. 122, Springer-Verlag, Berlin, 2006.

6. Jal R. Choksi and Mahendra G. Nadkarni, Baire category in spaces of measures, unitary operators, and transformations, Invariant Subspaces and Allied Topics, Narosa Publ. Co., New Delhi, 1990, pp. 147-163.

7. Ilijas Farah, A dichotomy for the Mackey Borel structure, Proceedings of the 11th Asian Logic Conference, World Scientific Publishing, Hackensack, NJ, 2012, pp. 86-93.

8. Ilijas Farah, Andrew S. Toms, and Asger Törnquist, Turbulence, orbit equivalence, and the classification of nuclear $C^{*}$-algebras, Journal für die Reine und Angewandte Mathematik 688 (2014), 101-146.

9. Matthew Foreman and Benjamin Weiss, An anti-classification theorem for ergodic measure preserving transformations, Journal of the European Mathematical Society 6 (2004), no. 3, 277-292.

10. Harvey Friedman and Lee Stanley, A Borel reducibility theory for classes of countable structures, Journal of Symbolic Logic 54 (1989), no. 3, 894-914.

11. Su Gao, On automorphism groups of countable structures, Journal of Symbolic Logic 63 (1998), no. 3, 891-896.

12. __ Invariant descriptive set theory, Pure and Applied Mathematics (Boca Raton), vol. 293, CRC Press, Boca Raton, FL, 2009.

13. Michael Hartz and Martino Lupini, The classification problem for operator algebraic varieties and their multiplier algebras, Transactions of the American Mathematical Society, to appear.

14. Greg Hjorth, Non-smooth infinite-dimensional group representations, (1997), unpublished note.

15. _ An absoluteness principle for Borel sets, The Journal of Symbolic Logic 63 (1998), no. 2, 663-693.

16. Classification and orbit equivalence relations, Mathematical Surveys and Monographs, vol. 75, American Mathematical Society, Providence, RI, 2000. 
17. Greg Hjorth, Alexander S. Kechris, and Alain Louveau, Borel equivalence relations induced by actions of the symmetric group, Annals of Pure and Applied Logic 92 (1998), no. 1, 63-112.

18. Greg Hjorth and Sławomir Solecki, Vaught's conjecture and the Glimm-Effros property for Polish transformation groups, Transactions of the American Mathematical Society 351 (1999), no. 7, 2623-2641.

19. Adrian Ioana, Alexander S. Kechris, and Todor Tsankov, Subequivalence relations and positive-definite functions, Groups, Geometry, and Dynamics 3 (2009), no. 4, 579-625.

20. Vladimir Kanovei, Borel equivalence relations, University Lecture Series, vol. 44, American Mathematical Society, Providence, RI, 2008, Structure and classification.

21. Alexander Kechris and Robin Tucker-Drob, The complexity of classification problems in ergodic theory, Appalachian Set Theory 2006-2012, London Math. Soc. Lecture Note Ser., vol. 406, Cambridge Univ. Press, Cambridge, 2012.

22. Alexander S. Kechris, Classical descriptive set theory, Graduate Texts in Mathematics, vol. 156, Springer-Verlag, New York, 1995.

23. Actions of Polish groups and classification problems, Analysis and logic (Mons, 1997), London Math. Soc. Lecture Note Ser., vol. 262, Cambridge University Press, Cambridge, 2002, pp. 115-187.

24. Global aspects of ergodic group actions, Mathematical Surveys and Monographs, vol. 160, American Mathematical Society, Providence, RI, 2010.

25. Alexander S. Kechris and Nikolaos E. Sofronidis, A strong generic ergodicity property of unitary and self-adjoint operators, Ergodic Theory and Dynamical Systems 21 (2001), no. 5, 1459-1479.

26. David Kerr, Hanfeng Li, and Mikaël Pichot, Turbulence, representations, and trace-preserving actions, Proceedings of the London Mathematical Society 100 (2010), no. 2, 459-484.

27. David Kerr, Martino Lupini, and N. Christopher Phillips, Borel complexity and automorphisms of C*-algebras, Journal of Functional Analysis 268 (2015), no. 12, 3767-3789.

28. Martino Lupini, Polish groupoids and functorial complexity, Transactions of the American Mathematical Society, in press.

29. Unitary equivalence of automorphisms of separable $C^{*}$-algebras, Advances in Mathematics 262 (2014), $1002-1034$.

30. Maciej Malicki, On Polish groups admitting a compatible complete left-invariant metric, Journal of Symbolic Logic $\mathbf{7 6}$ (2011), no. $2,437-447$.

31. Julien Melleray and Todor Tsankov, Generic representations of abelian groups and extreme amenability, Israel Journal of Mathematics 198 (2013), no. 1, 129-167.

32. Alan L. T. Paterson, Groupoids, inverse semigroups, and their operator algebras, Progress in Mathematics, vol. 170, Birkhäuser Boston Inc., Boston, MA, 1999.

33. Arlan B. Ramsay, The Mackey-Glimm dichotomy for foliations and other Polish groupoids, Journal of Functional Analysis 94 (1990), no. 2, 358-374.

34. , Polish groupoids, Descriptive set theory and dynamical systems, London Math. Soc. Lecture Note Ser., vol. 277, Cambridge University Press, Cambridge, 2000, pp. 259-271.

35. Román Sasyk and Asger Törnquist, Borel reducibility and classification of von Neumann algebras, Bulletin of Symbolic Logic 15 (2009), no. 2, 169-183.

36. Román Sasyk and Asger Törnquist, The classification problem for von Neumann factors, Journal of Functional Analysis 256 (2009), no. 8, 2710-2724.

37. Román Sasyk and Asger Törnquist, Turbulence and Araki-Woods factors, Journal of Functional Analysis 259 (2010), no. 9, $2238-2252$.

38. Asger Törnquist and Martino Lupini, Set theory and von Neumann algebras, Appalachian Set Theory 2006-2012, London Math. Soc. Lecture Note Ser., vol. 406, Cambridge Univ. Press, Cambridge, 2012.

Mathematics Department, Caltech, 1200 E. California Blvd, MC 253-37 Pasadena, CA 91125

E-mail address: lupini@caltech.edu

URL: http://www.lupini.org/

Department of Mathematics, 1409 W. Green St., University of Illinois, Urbana, IL 61801

E-mail address: panagio2@illinois.edu

$U R L:$ http://math.illinois.edu/ panagio2/ 